\title{
Study of the surface tension of polymer solutions : theory and experiments in theta solvent conditions
}

\author{
J. M. di Meglio, R. Ober, L. Paz, C. Taupin, P. Pincus $\left(^{++}\right)$ \\ Laboratoire de Physique de la Matière Condensée (*), Collège de France,
} 11, Place Marcelin-Berthelot, 75231 Paris Cedex 05, France

and S. Boileau

Laboratoire de Chimie Macromoléculaire (**), Collège de France, 11, Place Marcelin-Berthelot, 75231 Paris Cedex 05, France

(Reçu le 24 février 1983, accepté le 30 mai 1983)

\begin{abstract}
Résumé. - La tension superficielle de solutions semidiluées de polymères en solvant théta a été mesurée. Des prédictions théoriques sont faites pour les deux cas d'interface attractif ou répulsif. On utilise l'approche de Cahn pour le calcul de la tension interfaciale à l'aide de la théorie champ moyen des solutions de polymère. Les mesures ont été effectuées sur deux systèmes en conditions $\theta$ : polydiméthylsiloxane-bromocyclohexane à $29^{\circ} \mathrm{C}$ et polystyrène-cyclohexane à $34^{\circ} \mathrm{C}$ respectivement pour les cas attractif et répulsif. La méthode de l'anneau est utilisée. L'accord théorie-expérience est bon. Les prédictions théoriques et les résultats sont comparés à ceux obtenus dans le cas bon solvant lors d'une étude précédente. La transition $\theta$ solvant-bon solvant est étudiée pour le système attractif.
\end{abstract}

\begin{abstract}
A study of the surface tension of semi-dilute polymer solutions in theta solvent conditions is presented. Theoretical predictions are given for the two cases when the interface is attractive or repulsive. The Cahn approach for the interfacial tension is used in order to calculate the interfacial tension in the mean field theory of polymer solutions. The measurements, were performed on two polymer theta solvent systems : polydimethylsiloxanebromocyclohexane at $29^{\circ} \mathrm{C}$ and polystyrene-cyclohexane at $34^{\circ} \mathrm{C}$ for the attractive and repulsive cases respectively. The well-known ring method was used. The agreement between theory and experiments is good. The theoretical predictions and the results are compared with those obtained in good solvent case that we reported.in a previous paper. The transition $\theta$ solvent-good solvent is studied for the attractive system.
\end{abstract}

\section{Introduction.}

For a long time much effort has been devoted to the study of the interfacial properties of solutions. Recently, new theoretical studies [1-10] on these interfacial properties, in particular for the behaviour of polymer at liquid interfaces or free surfaces (melt or solution) have been developed and have raised new interest for experimental measurements.

In a previous paper [11], the surface tension of semi-dilute polymer solutions in good solvent condi-

(*) Equipe de Recherche Associée au C.N.R.S. (no 542).

(**) Associé au C.N.R.S.

$\left(^{++}\right)$Permanent address : Exxon Research, P.O. Box 45, Linden, N.J. 07036, USA. tions was examined both experimentally and theoretically. An attractive (polydimethylsiloxane (PDMS)toluene) and a repulsive (polystyrene (PS)-toluene) interface were investigated.

This paper presents both experimental and theoretical results for the $\theta$ solvent conditions. On the contrary to the good solvent case where several data exist for low molecular weight $\left(<10^{4}\right)$ polymers in various solvents [12-14], only one experimental study [12] $\left(M_{\mathrm{w}}<10^{4}\right)$ refers to $\theta$ conditions. We present here the first study of high molecular weight $\left(>3 \times 10^{5}\right)$ polymers on attractive and repulsive interfaces.

In the past few years very few theories $[5,12]$ consider the $\theta$ solvent conditions. The situation is presently changing since the approach of J. Cahn [7] for the interfacial tension and wetting properties of 
fluid-solid interface and some recent calculations $[8,9,15,16]$ of the profiles of polymer concentration in the vicinity of attractive or repulsive interfaces seem good building blocks for new prediction of interfacial behaviour.

We use a mean field theory, which is particularly valid in $\theta$ conditions and has serious advantages : the surface tension is given in analytical form and gives rise to numerical predictions and comparison between good solvent and $\theta$ solvent conditions. This theory indeed describes the essential experimental findings. The contribution of the very recent scaling theories $[17,18]$ are discussed in view of the good solvent $-\theta$ solvent transition.

\section{Experimental part.}

1.1 Chemical Systems. - We present experimental results in the semi-dilute range where the physical behaviour is independent of mass and polymer polydispersity. To make sure of the semidilute regime, we have used high molecular weight $\left(>3 \times 10^{5}\right)$ polymers since the lower limit volume fraction $\left(\Phi^{*}\right)$ varies as $M^{-0.5}$ in the $\theta$ solvent case instead of $M^{-0.8}$ in the good solvent case [19].

Two different polymers were used, polystyrene (PS) for the repulsive case and polydimethylsiloxane (PDMS) for the attractive one. The $\theta$ solvents were respectively cyclohexane $\left(\theta_{\mathrm{PS}}=34.5^{\circ} \mathrm{C}\right)$ [20] and bromocyclohexane $\left(\theta_{\mathrm{PDMS}}=28^{\circ} \mathrm{C}\right)$ [21]. Cyclohexane was purchased from Merck (Uvasol quality) and bromocyclohexane, purchased from Merck (zur Synthese) was redistilled under reduced pressure (20 torr) before use.

1.1.1. - One sample of polystyrene was used $\left(M_{\mathrm{w}}=426500, M_{\mathrm{n}}=322000, I_{\mathrm{p}}=1.32\right)$, purchased from EAHP (Strasbourg, France). In order to calculate the volume fraction of polymer in solution, we used the following values for the densities at $35^{\circ} \mathrm{C}$ :

$\rho_{\mathrm{PS}}=1.0753 \mathrm{~g} / \mathrm{cm}^{3} \quad[22] \quad \rho_{\text {cycl }}=0.765 \mathrm{~g} / \mathrm{cm}^{3}$

All the solutions were prepared by weighing polymer and solvent. The solubilization was performed in an oven at $54^{\circ} \mathrm{C} \simeq \theta+20^{\circ} \mathrm{C}$ during one week at least.

When examined by light scattering, the solutions revealed no aggregates, thus indicating a good solubilization.

1.1.2. - Two samples of polydimethylsiloxane (PDMS) were used (Table I), from the same origin as in [11].

Table I. - Molecular weights of the PDMS samples.

$\begin{array}{crrc}\text { Polymer } & M_{\mathrm{n} \mathrm{GPC}} & M_{\mathrm{w} \mathrm{GPC}} & I_{\mathrm{p} \mathrm{GPC}} \\ \text { I } & 54000 & 170000 & 3,15 \\ \text { III } & 414000 & 616000 & 1,49\end{array}$

The values of the densities were measured in the laboratory on a PAAR DMA 45 apparatus at $29^{\circ} \mathrm{C}$ :

$$
\rho_{\mathrm{BrCy}}=1.3257 \mathrm{~g} / \mathrm{cm}^{3}, \quad \rho_{\text {PDMS }}=0.9753 \mathrm{~g} / \mathrm{cm}^{3} \text {. }
$$

The solubilization was performed at $49{ }^{\circ} \mathrm{C}$ during at least one week for the first solubilization and at least 24 hours for the further dilutions.

1.2 Surface tension measurements. - We used the well-known-ring method [24-25]. The measurements were performed at $34{ }^{\circ} \mathrm{C}$ for the polystyrenecyclohexane system and at $29^{\circ} \mathrm{C}$ for the polydimethylsiloxane-bromocyclohexane system. The temperature was controlled within a range of $0.05{ }^{\circ} \mathrm{C}$. The apparatus was a modified Lauda tensiometer as described in [11].

Because of the longer kinetics of equilibrium for $\theta$ systems, after the solubilization stage in the oven at $\theta+20^{\circ} \mathrm{C}$, we transfer the sample in the tensiometer and let the solutions equilibrate at $\theta$ temperature during at least one night.

The measurement of the tension is given for each experiment within $0.02 \mathrm{mN} / \mathrm{m}$ and the reproducibility is between 0.05 and $0.1 \mathrm{mN} / \mathrm{m}$.

Repulsive interface.

For each concentration of the solution, at least, two measurements were performed. The surface tension of the cyclohexane at $34{ }^{\circ} \mathrm{C}$ was determined :

$$
\gamma_{0}=23.80 \pm 0.05 \mathrm{mN} / \mathrm{m} \text {. }
$$

The $\theta$ range was calculated following reference 26 to be $\theta \pm 5^{\circ} \mathrm{C}$. The concentration range was limited to $\Phi_{\mathrm{b}}=0.15$ since above this concentration, the viscoelastic properties of the surface increased the duration of the measurement so that some evaporation of the solvent occurred on the film leading to unreproducible results.

Attractive interface.

The surface tension of bromocyclohexane at $29^{\circ} \mathrm{C}$ was determined to be $\gamma_{0}=33 \pm 0.05 \mathrm{mN} / \mathrm{m}$.

The $\theta$ range is around $4{ }^{\circ} \mathrm{C}$ for sample III and $12^{\circ} \mathrm{C}$ for sample I [26].

\section{Theory. Comparison with the good solvent case.}

Following the Cahn approach [7], our starting point is to write the surface energy $\gamma$ as :

$$
\begin{aligned}
\gamma-\gamma_{0}=\gamma_{1} \Phi_{\mathrm{s}} & +\int_{0}^{\infty} \mathrm{d} z\{F(\Phi)- \\
& \left.-\mu_{\mathrm{b}} \Phi+\pi_{\mathrm{b}}+L(\Phi)\left(\frac{\mathrm{d} \Phi}{\mathrm{d} z}\right)^{2}\right\}
\end{aligned}
$$

where $\gamma_{0}$ is the surface tension of the pure solvent, the $z$ direction is normal to the surface and measured 
into the solution, $F(\Phi)$ is the free energy density for a semi-dilute polymer solution of volume fraction $\Phi$, $\mu_{\mathrm{b}}\left(=\left.\frac{\partial F}{\partial \Phi}\right|_{\Phi_{\mathrm{b}}}\right)$ is the chemical potential,

$$
\pi_{\mathrm{b}}\left(=\left.\Phi_{\mathrm{b}}^{2} \frac{\partial}{\partial \Phi}\left(\frac{F}{\Phi}\right)\right|_{\Phi_{\mathrm{b}}}\right)
$$

is the bulk osmotic pressure, $L(\Phi)$ describes the solution stiffness to spatial fluctuations of the concentration and $\gamma_{1}$ is a local solute-interface interaction energy/area which describes the attractive $\left(\gamma_{1}<0\right)$ or repulsive character $\left(\gamma_{1}>0\right)$ of the interface $\left(\Phi_{s}\right.$ is the polymer volume fraction at the interface). The integral term represents the energy change associated with the deformation of the concentration profile in the vicinity of the surface. The $\theta$ temperature corresponds to a tricritical point, so we expect that (to within logarithmic corrections) the mean field approximation is adequate. In mean field theory, the Flory-Huggins free energy density for semi-dilute solutions is [27] :

$$
F(\Phi)=\left(T / a^{3}\right)\left\{(\Phi / N) \operatorname{Ln} \Phi+\frac{1}{2} v \Phi^{2}+\frac{1}{6} w \Phi^{3}+\cdots\right\}
$$

where a is a monomer dimension, $N$ is the degree of polymerization and $T$ is the temperature measured in energy units. The first term represents the translational entropy of a polymer chain; $v$ and $w$ are, respectively, the second and third virial coefficients associated with the monomer-monomer interactions. For semi-dilute solutions the first term is usually neglected. This point could be questioned in the repulsive case where the volume fraction in the depletion layer can be less than the overlap volume fraction $\Phi^{*}$. But the thickness of the depletion layer is found experimentally to be close to $R_{\mathrm{G}}$ [28] and at this length scale, the translational entropy may be ignored; the interfacial energy is thus mainly dominated by the repulsive and gradient terms [27]. On the other hand there may be some limitation for the mean field approach in the adsorption layer where the concentration can be larger than $\Phi^{*}$. We can then follow the steps of our previous calculations but setting $v=0$ and keeping the third coefficient $1 / 6 w \Phi^{3}$. For the semi dilute regime the stiffness function $L(\Phi)$ in mean field theory is [27] :

$$
L(\Phi)=\left(\frac{a^{2}}{24 \Phi}\right) \frac{T}{a^{3}} .
$$

The interfacial energy then becomes :

$$
\begin{aligned}
\gamma-\gamma_{0}=\gamma_{1} \Phi_{\mathrm{s}}+\int_{0}^{\infty} \mathrm{d} z\{ & \frac{1}{6} w\left(\Phi^{3}-3 \Phi \Phi_{\mathrm{b}}^{2}+2 \Phi_{\mathrm{b}}^{3}\right)+ \\
& \left.+\frac{a^{2}}{24 \Phi}\left(\frac{\mathrm{d} \Phi}{\mathrm{d} z}\right)^{2}\right\} \frac{T}{a^{3}}
\end{aligned}
$$

Setting $y^{2}=\Phi / \Phi_{\mathrm{b}}, x=z / \xi_{\mathrm{b}}$ with $\xi_{\mathrm{b}}$ the Edwards correlation length [29]

$$
\xi_{\mathrm{b}}=a /\left(w^{1 / 2} \Phi_{\mathrm{b}}\right) \text { and } \sigma_{\mathrm{m}}^{-1}=\left(T / 6 \gamma_{1} a\right)\left(1 / \xi_{\mathrm{b}}\right)
$$

a dimensionless mean field adsorption constant, the surface energy is conveniently written :

$$
\frac{\gamma-\gamma_{0}}{\gamma_{1} \Phi_{\mathrm{b}}}=y_{\mathrm{s}}^{2}+\sigma_{\mathrm{m}}^{-1} \int_{0}^{\infty} \mathrm{d} x\left\{\left(y^{2}-1\right)^{2}\left(y^{2}+2\right)+\left(\frac{\mathrm{d} y}{\mathrm{~d} x}\right)^{2}\right\} \text {. }
$$

For fixed $y_{\mathrm{s}}$, the Euler-Lagrange equation derived from 2.4 is

$$
\frac{\mathrm{d}^{2} y}{\mathrm{~d} x^{2}}=3 y\left(y^{2}-1\right)\left(y^{2}+1\right)
$$

which has a first integral

$$
\frac{\mathrm{d} y}{\mathrm{~d} x}=\left(1-y^{2}\right)\left(y^{2}+2\right)^{1 / 2}
$$

which satisfies the boundary condition $\mathrm{d} \Phi / \mathrm{d} x=0$ as $\Phi \rightarrow \Phi_{b}$. Reinserting $d y / d x$ into (2.4), we obtain (as in [11]) :

$$
\frac{\gamma-\gamma_{0}}{\gamma_{1} \Phi_{\mathrm{b}}}=y_{\mathrm{s}}^{2}+2 \sigma_{\mathrm{m}}^{-1} \int_{0}^{\infty}\left(\frac{\mathrm{d} y}{\mathrm{~d} x}\right)^{2} \mathrm{~d} x
$$

or

$$
=y_{\mathrm{s}}^{2}+2 \sigma_{\mathrm{m}}^{-1} \int_{y_{\mathrm{s}}}^{1}\left(\frac{\mathrm{d} y}{\mathrm{~d} x}\right) \mathrm{d} y
$$

since for $x=0, y=y_{\mathrm{s}}$ and for $x=\infty, y=1$.

Dealing with $\theta$ solvents, we assumed to have a strong adsorption, i.e. $\left|\sigma_{\mathrm{m}}\right|>1, y_{\mathrm{s}}^{2} \gg 1$ for the attractive case and $y_{\mathrm{s}}^{2} \ll 1$ for the repulsive case.

The interfacial free energy is then minimized by

$$
\sigma_{\mathrm{m}} y_{\mathrm{s}}=\left(\frac{\mathrm{d} y}{\mathrm{~d} x}\right)_{y_{\mathrm{s}}}=\left(1-y_{\mathrm{s}}^{2}\right)\left(y_{\mathrm{s}}^{2}+2\right)^{1 / 2}
$$

letting in the attractive case

$$
\begin{aligned}
& y_{\mathrm{s}}^{2} \simeq\left|\sigma_{\mathrm{m}}\right| \text { or more explicitely } \\
& \Phi_{\mathrm{s}}=6\left(\frac{\left|\gamma_{1}\right| a^{2}}{T}\right) w^{-1 / 2}
\end{aligned}
$$

and in the repulsive case

$$
y_{\mathrm{s}}^{2} \simeq 2 \sigma_{\mathrm{m}}^{-2} \text { or } \Phi_{\mathrm{s}}=\left(\frac{\sqrt{2}}{6} \frac{T}{\gamma_{1} a^{2}} w^{1 / 2}\right) \Phi_{\mathrm{b}}^{3} .
$$


The interfacial tension is then easily calculated knowing $y_{\mathrm{s}}$ from (2.7) leading :

- in the attractive case $\left(\sigma_{\mathrm{m}}<0,\left|\sigma_{\mathrm{m}}\right|>1, y>1\right.$, $\left.\gamma_{1}<0\right)$

$$
\begin{aligned}
\gamma-\gamma_{0}=3 \gamma_{1} & \left(\frac{\left|\gamma_{1}\right| a^{2}}{T}\right) w^{-1 / 2}- \\
& -\left(\frac{T w^{1 / 2}}{4 a^{2}}\right) \Phi_{\mathrm{b}}^{2} \log \frac{6\left|\gamma_{1}\right| a^{2}}{T w^{1 / 2} \Phi_{\mathrm{b}}}
\end{aligned}
$$

- in the repulsive case $\left(\sigma_{\mathrm{m}}>0, \sigma_{\mathrm{m}} \gg 1, y<1\right)$

$$
\gamma-\gamma_{0}=\frac{\sqrt{2}}{6}\left(\frac{T w^{1 / 2}}{a^{2}}\right) \Phi_{\mathrm{b}}^{2} .
$$
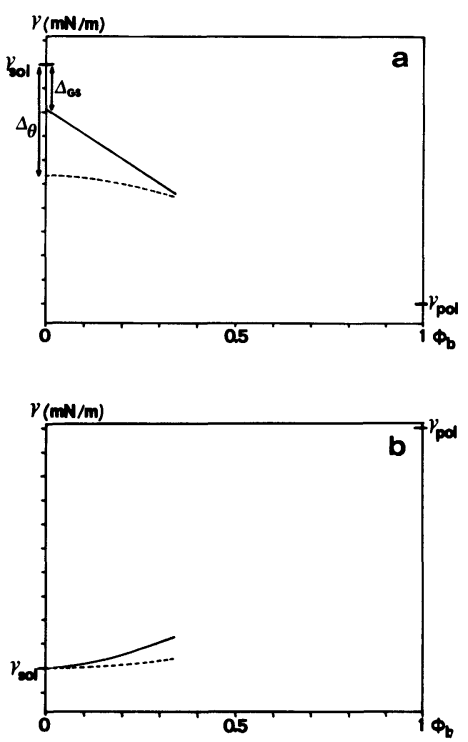

Fig. 1. - Theoretical variation of the surface tension of polymer solution in good solvent (solid line) and $\theta$ solvent conditions (dotted line). a) attractive case; $b$ ) repulsive case. The following numerical values were taken $: \mid \gamma_{\text {sol }}-$ $\gamma_{\text {pol }} \mid=10 \mathrm{mN} / \mathrm{m}, \gamma_{1}=5 \mathrm{mN} / \mathrm{m}, a=5 \AA, v=1$ (G.S.), $w=1\left(\theta \mathrm{S}\right.$.) corresponding to a coupling constant $\gamma_{1} a^{2} / T$ equal to 0.31 .

These equations are plotted on figure 1 together with those which were obtained in the good solvent case. We can point out two remarks :

2.1 AtTRACTIVE INTERFACE. - For a given value of the coupling constant $\left(\gamma_{1} a^{2} / T\right)$ which is always smaller than 1, the sharp drop at $\Phi_{\mathbf{b}} \rightarrow 0$ of the surface tension is larger for the $\theta$ solvent $\left(\Delta \gamma / \gamma_{1} \sim\left(\gamma_{1} a^{2} / T\right)\right)$ than for the good solvent where $\left(\Delta \gamma / \gamma_{1} \sim\left(\gamma_{1} a^{2} / T\right)^{2}\right):$ the weaker repulsive interactions between monomers allow a denser adsorption layer in the $\theta$ solvent case. The variation of the surface tension is linear in $\Phi_{\mathrm{b}}$ for good solvent and quadratic in $\Phi_{\mathrm{b}}$ for the $\theta$ solvent with a coefficient of the same order of magnitude. As $\Phi_{\mathrm{b}}$ is much smaller than 1 , this leads to smaller variations of $\gamma$ for the $\theta$ solvent than for the good solvent (in the semi-dilute concentration range).
2.2 RePUlSIVE INTERFACE. - In comparison with the good solvent case, we find a change in the exponent of the concentration dependence varying from $3 / 2$ in the good solvent to 2 in the $\theta$ solvent. The coefficients of $\Phi_{b}^{3 / 2}$ and $\Phi_{\mathrm{b}}^{2}$ are of the same order of magnitude, and we expect a smaller variation of $\gamma$ for the $\theta$ solvent than for the good solvent in the $\Phi_{b}$ concentration range.

2.3 COMPARISON WITH EARLIER THEORIES. - Earlier theories $[12,13,30]$ are monolayers theories which predict an adsorption or depletion layer but no profile of concentrations. These theories describe correctly the attractive case where surface tension is determined by the adsorbed species but they do not lead to satisfactory results for the repulsive case, and for high polymers, the meaning of the results is even doubtful [13] $(\gamma<0)$. Moreover they do not follow the Gibbs equation [31]. Poser and Sanchez theory [10] is similar to our one except that their gradient coefficient is kept constant while a mean field theory predicts a $\Phi^{-1}$ dependence $[27,32]$.

\section{Results and discussion.}

We measured the surface tension of polymer solutions in a wide range of volume concentration up to 0.15-0.25.

To our knowledge, only one study [12] refers to measurements of the surface tension of polymer solutions in $\theta$ conditions. The authors studied both attractive and repulsive systems. In the attractive case, PS in cyclohexane at the water-cyclohexane interface was used. A discrepancy between theory and experiment was observed which could be due to the low molecular weight $\left(<10^{4}\right)$ of the samples which are not the best candidates to test polymer theories. The repulsive system was : polyisobutylene of $M_{\mathrm{w}}$ around $10^{3}$ in benzene at the water-benzene interface. The comparison with theory leads to $\chi=0.2$ (Flory parameter) which corresponds rather to good solvent case.

The theoretical predictions are related to the semidilute concentration range. In fact, the expression semi-dilute range deserves some discussion : if all the authors agree on the definition of the lower limit (overlap concentration) the upper limit is not so well understood. Experimentally, the situation is not clear [33] and we consider that an upper limit of this range is 0.25 as in [11]. We evaluated the lower limit of the semi-dilute concentration range using reference 19. For PS with a molecular weight $M_{\mathrm{w}}=426500$, we obtain [26] a limit concentration $\Phi^{*}=0.065$ which is a high value and limits the semi-dilute range. For PDMS, from the hydrodynamic radius of chains measured in [34], we inferred a limit value $\Phi^{*}=0.063$ for PDMS I and $\Phi^{*}=0.035$ for PDMS III. Note that in the case of an attractive interface, the concentration near the interface is much higher than in 
bulk, thus extending the validity of semi-dilute calculation for $\Phi_{b}<\Phi^{*}$.

3.1 REPUlSIVE INTERFACE. - Figure 2 shows the variation of $\gamma$ as a function of the volumic fraction $\Phi_{b}$. The comparison with figure 3 of (11) shows that the general shape of the curve is the same namely $\gamma-\gamma_{0}=A \Phi^{\alpha}$ but that the variation of $\gamma$ with polymer concentration is approximately six times smaller than with good solvent.

The quantitative comparison with (2.10) gives $\gamma_{0}=23.8 \mathrm{mN} / \mathrm{m}$ and $w=0.2 \pm 0.1$. This value of $w$ is clearly a reasonable one $\left(^{1}\right)$.

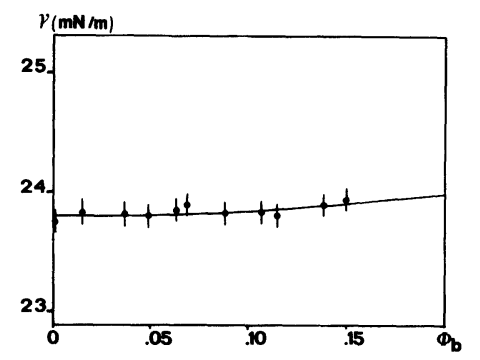

Fig. 2. - Repulsive case PS-cyclohexane $\left(35^{\circ} \mathrm{C}\right)$. Plot of $\gamma$ as a function of $\Phi_{\mathrm{b}}$. The curve corresponds to the equation 2.10. A linear plot in $\Phi_{b}$ was chosen since a plot on $\Phi_{b}^{2}$ would superimpose most of the experimental points due to the small variation of $\gamma$.

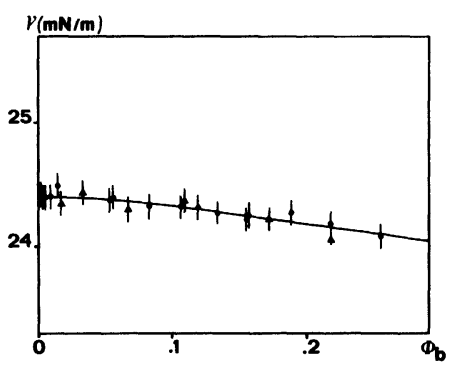

Fig. 3. - Attractive case PDMS-Bromocyclohexane (29 ${ }^{\circ} \mathrm{C}$ ). Plot of $\gamma$ as a function of $\Phi_{b}$. The curve corresponds to the equation $2.8\left(\bullet M_{\mathrm{w}}=170000, \Delta M_{\mathrm{w}}=616000\right)$. A linear plot $\Phi_{\mathrm{b}}$ was chosen since a plot on $\Phi_{\mathrm{b}}^{2}$ would superimpose most of the experimental points due to the small variation of $\gamma$.

3.2 Attractive INTERFACE. - The comparison of figure 3 with figure 4 of the previous paper shows that, like in the repulsive case, the general shape of the curve is the same namely :

- no dependence on molecular weight of the polymer,

- a sharp drop from $33 \mathrm{mN} / \mathrm{m}$ to $24.4 \mathrm{mN} / \mathrm{m}$ at very low concentration $\left(\Phi_{\mathrm{b}}<0.001\right)$,

- then a progressive decrease of $\gamma$; the curvature is more pronounced than in the good solvent case,

- the overall variation of $\gamma$ is also five times smaller than in the good solvent case.

( ${ }^{1}$ ) Recent unpublished measurements by Mc. Donald (West Virginia Univ.) give $w=0.28$.
Following (2.8), the best fit shown on figure 3 gives :

$$
\begin{aligned}
\gamma_{0} & =33 \mathrm{mN} / \mathrm{m} \\
\left|\gamma_{1}\right| & =4.8 \pm 0.2 \mathrm{mN} / \mathrm{m} \\
w & =0.75 \pm 0.20 .
\end{aligned}
$$

The value of $\gamma_{1}$ which is related with the surface tension of the two materials is coherent with the findings of [11] where we got

$$
\left|\gamma_{1}\right|=2 \mathrm{mN} / \mathrm{m} \text { with } \gamma_{\text {Tol }}-\gamma_{\text {Pol }} \sim 5 \mathrm{mN} / \mathrm{m}
$$

and here

$$
\left|\gamma_{1}\right|=4.8 \mathrm{mN} / \mathrm{m} \text { with } \quad \gamma_{\mathrm{BrC}}-\gamma_{\mathrm{Pol}} \sim 13 \mathrm{mN} / \mathrm{m} \text {. }
$$

The value of $w$, of the order of unity [27] is reasonable.

\section{Transition $\theta$ solvent-good solvent. Attractive case.}

Let us consider the two meanfield expressions of the surface tension in good solvent (G.S.) and $\theta$ solvent $(\theta$ S.) :

$$
\begin{gathered}
\gamma_{\mathrm{GS}}-\gamma_{0}=\gamma_{1}\left[\frac{4}{v}\left(\frac{\gamma_{1} a^{2}}{T}\right)^{2}+2 \Phi_{\mathrm{b}}\right] \\
\gamma_{\theta}-\gamma_{0}=\gamma_{1}\left[-\frac{3 \gamma_{1} a^{2}}{T w^{1 / 2}}-1 / 4 \frac{T w^{1 / 2}}{\gamma_{1} a^{2}} \Phi_{\mathrm{b}}^{2} \operatorname{Ln} \frac{6 \gamma_{1} a^{2}}{T w^{1 / 2} \Phi_{\mathrm{b}}}\right] .
\end{gathered}
$$

Each of them depend only on two parameters $\gamma_{1}$ and either $\left(\frac{\gamma_{1} a^{2}}{v^{1 / 2} T}\right)$ or $\left[\frac{\gamma_{1} a^{2}}{w^{1 / 2} T}\right]$ for good and $\theta$ conditions respectively. Moreover these parameters are not correlated with each other in the fit. As a consequence, the study of the G.S. $\theta$ S. transition on the same polymer-solvent system gives $\gamma_{1}$ as a function of the quality of the solvent. Moreover the fact that $a$ is identical in both conditions permits a better check of theoretical formula.

We measured the surface tension of PDMS-bromocyclohexane and of pure bromocyclohexane in the $25-55^{\circ} \mathrm{C}$ temperature range. One question appears : does $\theta+25^{\circ} \mathrm{C}$ correspond to G.S. conditions ? The vicinity of the $\theta$ point was studied by means of neutron-scattering by Cotton et al. [35]. If we consider their (concentration-temperature) diagram as a typical example, the transition from $\theta$ region to good solvent region in the semidilute range $\left(\Phi_{b} \sim 0.1\right)$ would occur at $\theta+5^{\circ} \mathrm{C}$. Their experimental data on the radius of gyration of $2 \times 10^{5} M_{\mathrm{w}}$ polymers indicate that the good solvent behaviour is observed at $\theta+20^{\circ} \mathrm{C}$.

As for a $6 \times 10^{5} M_{\mathrm{w}}$ polymer (polymer III) the transition is sharper so that we infer $\theta+25^{\circ} \mathrm{C}$ to be good solvent conditions even with the polydispersity index of 1.5 .

Table II compares the drop of the surface tension at $\Phi_{\mathrm{b}} \rightarrow O\left(\Delta \gamma_{0}\right)$ and the dependence of $\gamma$ on $\Phi_{\mathrm{b}}$ at $\theta$ and $\theta+25^{\circ} \mathrm{C}$ : the larger dependence of $\gamma$ on $\Phi_{\mathrm{b}}$ (by a factor of ten) at $55^{\circ} \mathrm{C}$ indicates G.S. conditions and gives $\left|\gamma_{1}\right|$ equal to $2.5 \mathrm{mN} / \mathrm{m}$ instead of $4.8 \mathrm{mN} / \mathrm{m}$ at $\theta$. 
Table II. - Comparison of various parameters for the PDMS-bromocyclohexane system.

$\begin{array}{lcccl} & & \begin{array}{c}\Delta \gamma_{0} \\ (\mathrm{mN} / \mathrm{m})\end{array} & \begin{array}{c}\gamma_{\Phi_{\mathrm{b}} \rightarrow 0}-\gamma_{\Phi_{\mathrm{b}}=0.1} \\ (\mathrm{mNN} / \mathrm{m})\end{array} & \begin{array}{c}\left|\gamma_{1}\right| \\ (\mathrm{mN} / \mathrm{m})\end{array} \\ \theta & 29{ }^{\circ} \mathrm{C} & 8.6 & 0.08 & 4.8 \\ \text { GS } & 55^{\circ} \mathrm{C} & 6.8 & 0.5 \pm 0.1 & 2.5 \pm 1.0\end{array}$

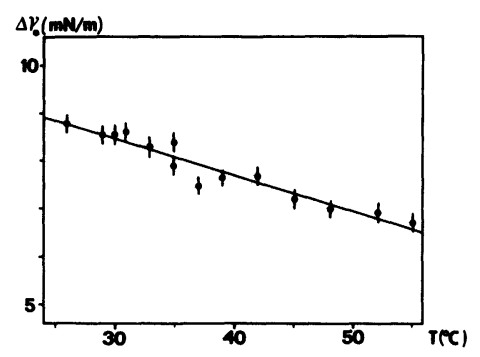

Fig. 4. - Variation of the drop of the surface tension at $\Phi_{b} \rightarrow 0\left(\Delta \gamma_{0}\right)$ as a function of temperature for the PDMSbromocyclohexane system.

Figure 4 shows the variation of $\Delta \gamma_{0}$ as a function of temperature of about $20 \%$ smaller than the corresponding variation of $\gamma_{1}(50 \%)$. It is difficult to draw more quantitative conclusions since we do not have information on $v$ in these conditions (PDMS-GS). Nevertheless, it is interesting to compare $\Delta \gamma_{0}$ in our two PDMS-GS systems, and a system measured by Gaines [13] (Table III).

Table III. - Result of various PDMS-GS solvent systems.

Toluene : $\quad \Delta \gamma_{0}=3 \mathrm{mN} / \mathrm{m} \quad\left|\gamma_{1}\right|=2 \mathrm{mN} / \mathrm{m}$ Bromocyclohexane : $\Delta \gamma_{0}=6.8 \mathrm{mN} / \mathrm{m} \quad\left|\gamma_{1}\right|=2.5 \mathrm{mN} / \mathrm{m}$ Tetrachloroethylene : $\Delta \gamma_{0}=4.4 \mathrm{mN} / \mathrm{m} \quad\left|\gamma_{1}\right|=3.2 \mathrm{mN} / \mathrm{m}$

If we consider the same $a$, characteristic of PDMS, and similar values of $v$, the mean field theory predicts $\Delta \gamma_{0} \alpha \gamma_{1}^{3}$. The values of table II suggest a lower dependence of $\Delta \gamma_{0}$ on $\gamma_{1}$. Recent papers $[17,18]$ based on scaling theory predict a weaker dependence of $\Delta \gamma_{0}$ on the solvent quality : in $\gamma_{1}^{2}$ both for $\theta$ and GS conditions. The continuity between GS and $\theta$ solvent conditions and the above values of table II suggest a better agreement of the experimental results with the scaling theories.

\section{Acknowledgments.}

We thank P. G. de Gennes who initiated this study.
[1] Cahn, J. W., Hilliard, J. E., J. Chem. Phys. 28 (1958) 258.

[2] Bongiorno, V., Davis, H. T., Phys. Rev. A 12 (1975) 2213.

[3] Vriu, A., Roeberson, G. J., J. Polymer Sci. 15 (1977) 109.

[4] Helfand, E., J. Chem. Phys. 56 (1972) 3592.

[5] Silberberg, A., J. Chem. Phys. 48 (1968) 2835.

[6] Roe, R. J., J. Chem. Phys. 60 (1974) 4192.

[7] Cahn, J. W., J. Chem. Phys. 66 (1977) 3667.

[8] Joanny, J. F., Leibler, L., De Gennes, P. G., J. Polymer Sci. Polym. Phys. 17 (1979) 1073.

[9] De Gennes, P. G., Macromolecules 14 (1981) 1637.

[10] Poser, C. I., SANCHez, I. C., Macromolecules 14 (1981) 361.

[11] Ober, R., Paz, L., Taupin, C., Pincus, P., BoILEAU, S., Macromolecules 16 (1983) 50.

[12] Siow, K. S., Patterson, D., J. Phys. Chem. 77 (1973) 356.

[13] Gaines, G. L., J. Phys. Chem. 73 (1969) 3143.

[14] Roe, R. J., J. Phys. Chem. 72 (1968) 2013.

[15] Richmond, P., Lal, M., Chem. Phys. Lett. 24 (1974) 594.

[16] JoNes, I. S., Richmond, P., J.C.S. Faraday Trans. II 73 (1977) 1062.

[17] De Gennes, P. G., Pincus, P., J. Physique Lett. 44 (1983) L-241.

[18] Eisenriegler, E., Kremer, K., Binder, K., J. Chem. Phys. 77 (1982) 6296.

[19] Daoud, M., Cotton, J. P., Farnoux, B., Jannink, G., Sarma, G., Benoit, H., Duplessix, R., Picot, C., DE GeNNES, P. G., Macromolecules 8 (1975) 804.
[20] Schultz, A. R., Flory, J. P., J. Am. Chem.Soc. 76 (1954) 3758.

[21] Haug, A., Meyerhoff, G., Makromol. Chem. 53 (1962) 91.

[22] Polymer Handbook, Brandup, J.; Immergut, E. H. ed. (John Wiley and Sons, 2nd Ed.) 1974, p. IV 131.

[23] Organic solvents vol. II Riddick, J. A.; Bunger, W. B. Techniques of Chemistry (ed. John Wiley and Sons, 3rd Ed.) 1970.

[24] Du Nouy, J., Gen. Physic 1 (1918) 521.

[25] Harkins, W. D., Jordan, H. F., J. Am. Chem. Soc. 52 (1930) 1751.

[26] Adam, M., Delsanti, M., J. Physique 41 (1980) 713.

[27] An excellent general reference is DE GENNES, P. G., "Scaling concepts in Polymer Physics " (Cornell University Press, Ithaca) 1979.

[28] Allain, C., Ausserre, D., Rondelez, F., Phys. Rev. Lett. 49 (1982) 1695.

[29] Edwards, S. F., J. Phys. A 8 (1975) 1670.

[30] Prigogine, I., Defay, R., J. Colloid Sci. 7 (1952).

[31] Defay, R., Prigogine, I., Tension superficielle et adsorption (Ed. Dosoer, Liège) 1951.

[32] Helfand, E., Sapse, A. M., J. Chem. Phys. 62 (1975).

[33] Graessley, W. W., Polymer 21 (1980) 258.

[34] Munch, J. P., Herz, J., Bolleau, S., Candau, S., Macromolecules 14 (1981) 1370.

[35] Cotton, J. P., Nierlich, H., Boue, F., Daoud, M., Farnoux, B., Jannink, G., Duplessix, R., Picot, C., J. Chem. Phys. 65 (1976) 1101. 